\title{
New Generation Sequencing in Cholangiocarcinoma and Gallbladder Cancer: Clinical Implications for Immunotherapy
}

\author{
Hao Song ${ }^{1,}$, Hongyan $\mathrm{Ma}^{2}$, Yao Huang ${ }^{1}$, Bin Yi ${ }^{1}$, Xiaoqing Jiang ${ }^{1}$ \\ ${ }^{1}$ Department of Organ Transplantation, Eastern Hepatobiliary Surgery Hospital Affiliated to Shanghai Second Military Medical University, \\ Shanghai, China \\ ${ }^{2}$ Pharmacy Department, Shenzhen Longhua District Central Hospital, Shenzhen, China
}

Email address:

Jxq1225@sina.com (Xiaoqing Jiang)

${ }^{*}$ Corresponding author

\section{To cite this article:}

Hao Song, Hongyan Ma, Yao Huang, Bin Yi, Xiaoqing Jiang. New Generation Sequencing in Cholangiocarcinoma and Gallbladder Cancer: Clinical Implications for Immunotherapy. International Journal of Clinical and Experimental Medical Sciences.

Vol. 7, No. 4, 2021, pp. 91-97. doi: 10.11648/j.ijcems.20210704.14

Received: June 20, 2021; Accepted: July 8, 2021; Published: July 16, 2021

\begin{abstract}
Objective: To explore the common mutation gene and mutation rule of cholangiocarcinoma and gallbladder cancer, and to confirm the therapeutic effect of PD1 inhibitor through follow-up. Method: From 2017 to 2019,42 patients with intrahepatic cholangiocarcinoma (ICCA), 49 patients with extrahepatic cholangiocarcinoma (ECCA) and 40 patients with GBC were followed up in Eastern Hepatobiliary Hospital for new generation sequencing (NGS). And we also followed up 25 cases of cholangiocarcinoma with PD1 inhibitor and observed the therapeutic effect. Result: The result show that TP53, KRAS, ARID1A, CDKN2A, SMAD4 and ERBB are the most common mutations in ICCA. TP53, KRAS, CDKN2A, ARID2, SMAD4 and ARID1A are the most common mutations in ECCA. TP53, ERBB, CDKN2A, ARID1A, CCNE1 are the most common mutations in GBC. 25 patients who used PD1 inhibitors had no complete remission, 6 patients had partial remission, 6 patients had no effect at all, and the disease progressed. The objective remission rate reached $24 \%$ and progression free survival was 5.2 \pm 4.8 months. Conclusion: TP53, ARID1A, CDKN2A are the most common mutations in CCA or GBC. We believe that through continuous clinical trials, we will find drugs to inhibit these mutations. PD-1 inhibitor can be used as a choice for patients with advanced stage of CCA or GBC, but not all of them have good therapeutic effect. It is suggested to combine other methods for treatment.
\end{abstract}

Keywords: Cholangiocarcinoma, Gallbladder Cancer, New Generation Gene Sequencing, PD-1 Inhibitor

\section{Introduction}

CCA and GBC are high degree of malignancy tumors. They have metastasized distantly through hepatoduodenal ligament lymph nodes. Surgical resection is the only way to cure $\mathrm{CCA}$ and $\mathrm{GBC}$, but about $60 \%$ of patients lose the chance of surgery and insensitive to conventional chemotherapy [1]. The 2 year survival rate of these inoperable patients was less than $10 \%$ [2]. In the past, our understanding of gene analysis and molecular level of cholangiocarcinoma was often limited to known oncogenes and tumor suppressor factors, which hindered the development of immunotherapy. CCA and GBC usually involves a variety of genetic changes, but only one or several genetic changes are responsible for tumorigenesis. These are known as "driver mutations" and the other "passenger mutations" [3]. This paper mainly discusses the characteristics of common mutation genes in CCA and GBC and the effect of PD1 inhibitor.

\section{Materials and Methods}

Patients with biliary tract tumor, including pathologically confirmed CCA and GBC, were eligible for our study. Informed consent was obtained for tumor profiling and targeted therapy following protocol 
approved by the Institutional Ethics Review Committee at Eastern Hepatobiliary Surgery Hospital (Shanghai, China). The study was conducted in accordance with the Declaration of Helsinki and Good Clinical Practice guidelines. All patients signed consent before participating in the research.

\subsection{Sample Collection and Targeted Next-Generation Sequencing}

Tumor samples were obtained from participants at different clinical stages. Detailed information of the samples is summarized in Table 1. All tumor tissues were reviewed by two independent pathologists before sample disposal to confirm the pathologic diagnoses. Macrodissection on tissue slides was performed to evaluate tumor content and percentage. For each tumor sample, at least 15 unstained slides containing more than $20 \%$ tumor cells, a formalinfixed paraffin-embedded (FFPE) block, or $0.5 \mathrm{~cm}^{3}$ freshfrozen tissue was required, to extract DNA for each sample. DNA extraction was performed, and 50-250 ng doublestranded DNA was interrupted ultrasonically. Comprehensive genomic alteration analysis of the tumor and matched blood samples were performed with an assay panel that captured 450 cancer-related genes and selected introns of 38 genes frequently rearranged in cancer (YuansuTM, OrigiMed). With a mean coverage of $900 \times$ for tumor tissues and $300 \times$ for paired blood cells by an Illumina NextSeq-500 Platform (Illumina Incorporated, San Diego, CA).

\subsection{Variations Calling}

Somatic alternations, including base substitutions, insertions and deletions, copy number alterations, and gene fusions/rearrangements, were identified. The bioinformatic protocol is similar with previous published study. Briefly, reads were aligned to human genome with the reference sequence (hg19) by Burrows-Wheeler Aligner (BWA), accompanying with removing duplicates from polymerase chain reaction (PCR) by Picard. MuTect identified SNVs and short indels after quality recalibration and realignment using Genome Analysis Toolkit (GATK) pipeline. Pindel was used to calibrate short indels. Read depths were normalized in target regions by Exome Copy number Alterations/Variations annotATOR (EXCATOR). Customized algorithms were used to detect copy number changes and gene rearrangement. For copy-number variations (CNVs), genes with a threshold of surpassing 4-copies are deemed as amplification, and genes with 0 -copies are homozygous deletions.

TMB was estimated for each sample by counting its somatic mutations including coding SNVs and indels per megabase. Driver mutations and germline alterations in dbSNP database were not counted, following the method of Chalmers et al.

Table 1. Clinical characteristics of the study population $(N=131)$.

\begin{tabular}{lllll}
\hline & ECCA (n=49) & ICCA (n=42) & GBC (n=40) & ALL (N=131) \\
\hline Age (mean, range) & $56.79(30 \sim 74)$ & $57.88(32 \sim 78)$ & $62(40 \sim 79)$ & $56.98(26 \sim 78)$ \\
Sex (male) & $36(73.46 \%)$ & $26(61.9 \%)$ & $15(37.5 \%)$ & $77(58.77 \%)$ \\
Clinical stage ( $\geq 3)$ & $17(73.21 \%)$ & $18(80 \%)$ & $10(25 \%)$ & $45(34.35 \%)$ \\
HBV/HCV infection & $7(12.5 \%)$ & $12(21.81)$ & $5(12.5 \%)$ & $24(18.32 \%)$ \\
Liver cirrhosis & $3(5.35 \%)$ & $8(14.54 \%)$ & $4(10 \%)$ & $15(11.45 \%)$ \\
Biliary stone disease & $4(7.14 \%)$ & $2(3.63 \%)$ & $13(32.5 \%)$ & $19(14.5 \%)$ \\
Tumor metastasis & $20(35.71 \%)$ & $20(36.36 \%)$ & $7(17.5 \%)$ & $47(35.87 \%)$ \\
Operation history & $14(25 \%)$ & $26(47.27)$ & $37(92.5 \%)$ & $77(58.77 \%)$ \\
Family cancer history & & & & $19(14.5 \%)$ \\
Yes & $10(17.85 \%)$ & $6(10.9 \%)$ & $3(7.5 \%)$ & $93(70.99 \%)$ \\
No & $31(21.42 \%)$ & $30(52.72 \%)$ & $32(80 \%)$ & $19(14.5 \%)$ \\
Unknown & $8(60.71 \%)$ & $6(36.36 \%)$ & $5(12.5 \%)$ & \\
\hline
\end{tabular}

\section{The Most Common Mutation Gene in Cholangiocarcinoma}

Variations in splicing and promoter regions of the gene can produce multiple transcriptional abnormalities and protein subtypes. Abnormal translation of the initial codon also results in more protein subtypes [4]. After analyzing the results of gene detection in 42 cases of ICCA, 49 cases of ECCA and 40 cases of GBC, we found that the most common gene mutations in intrahepatic cholangiocarcinoma were TP53, KRAS, ARID1A, CDKN2A, SMAD4 and ERBB; TP53, KRAS, CDKN2A, ARID2, SMAD4 and ARID1A are the most common mutations in extrahepatic cholangiocarcinoma. While TP53, ERBB, CDKN2A, PIK3CA, ARID1A, CCNE1 are the most common mutations in GBC (Figure 1, Table 2).

Table 2. Proportion of common mutations in CCA and GBC.

\begin{tabular}{lllll}
\hline & ICCA (42) & ECCA (49) & GBC (40) & Total (131) \\
\hline TP53 & $16(38.09 \%)$ & $24(48.97 \%)$ & $28(70 \%)$ & $68(51.9 \%)$ \\
KRAS & $14(33.33 \%)$ & $25(51.02 \%)$ & $3(7.5 \%)$ & $42(32.06 \%)$ \\
ARID1A & $12(28.57 \%)$ & $4(8.16 \%)$ & $6(15 \%)$ & $22(16.79 \%)$ \\
CDKN2A & $8(19.04 \%)$ & $8(16.32 \%)$ & $9(22.5 \%)$ & $24(18.32 \%)$ \\
ERBB & $7(16.66 \%)$ & $4(8.16 \%)$ & $10(25 \%)$ & $21(16.03 \%)$ \\
SMAD4 & $8(19.04 \%)$ & $5(10.2 \%)$ & $5(12.5 \%)$ & $18(13.74 \%)$ \\
ARID2 & $3(7.14 \%)$ & $6(12.24 \%)$ & $2(5 \%)$ & $11(8.39 \%)$ \\
ATM & $1(2.38 \%)$ & $5(10.2 \%)$ & $5(12.5 \%)$ & $11(8.39 \%)$ \\
CDK12 & $1(2.38 \%)$ & $3(6.12 \%)$ & $6(15 \%)$ & $10(7.63 \%)$ \\
CCNE1 & $3(7.14 \%)$ & $1(2.04 \%)$ & $6(15 \%)$ & $10(7.63 \%)$ \\
\hline
\end{tabular}




\begin{tabular}{|c|c|c|c|c|c|c|c|c|c|c|c|c|c|c|c|c|}
\hline Age & & Il & IIII & أ. & & & & & & & & 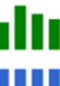 & . & & & In \\
\hline Cancer_type & & 11 & & & & & & & & & & & & & & III \\
\hline TP53 & $48 \%$ & I & & 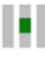 & ! & H & & 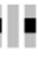 & & 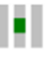 & H & 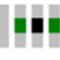 & $H$ & & H & nth \\
\hline KRAS & $35 \%$ & 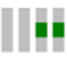 & H & $H$ & H & II & & I & 4 & 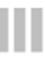 & H. & H & I & & & \\
\hline ARID1A & $23 \%$ & 11 & 1 & &  & & 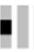 & ! & & - & 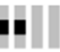 & . & 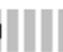 & ! & & 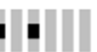 \\
\hline LRP1B & $19 \%$ & $H$ & H & & $H$ & & & & & & H & & & & & II \\
\hline SMAD4 & $15 \%$ & - & 1 & & H & ॥ & & & & & 1 & & 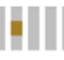 & & प & \\
\hline CDKN2A & $13 \%$ & & II & & | & & & & & & n & & & & & 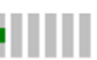 \\
\hline PBRM1 & $13 \%$ & $\|$ & 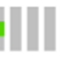 & & ! & & & | & & 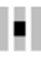 & 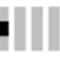 & & $\|$ & & & 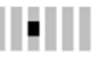 \\
\hline APC & $10 \%$ & & I & & 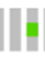 & & 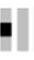 & & 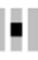 & & & - & 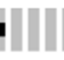 & & & 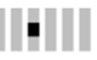 \\
\hline ARID2 & $10 \%$ & 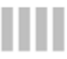 & 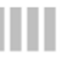 & & 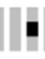 & प & & & & $H$ & & & & & & \\
\hline GLI3 & $10 \%$ & $\|$ & $\|$ & & H & & & & & H & $\|$ & 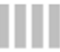 & & & & H \\
\hline
\end{tabular}

$\begin{array}{ll}\text { Genetic Alteration } & \text { Inframe Mutation (putative driver) } \| \text { Inframe Mutation (unknown significance) } \| \text { Missense Mutation (putative driver) } \| \text { Missense Mutation } \\ \text { Age } & \text { Truncating Mutation (putative driver) } \| \text { Truncating Mutation (unknown significance) } \| \text { No alterations } \\ \text { Cancer_type } & \text { cholangiocarcinoma } \quad \text { gallbladder_cancer }\end{array}$

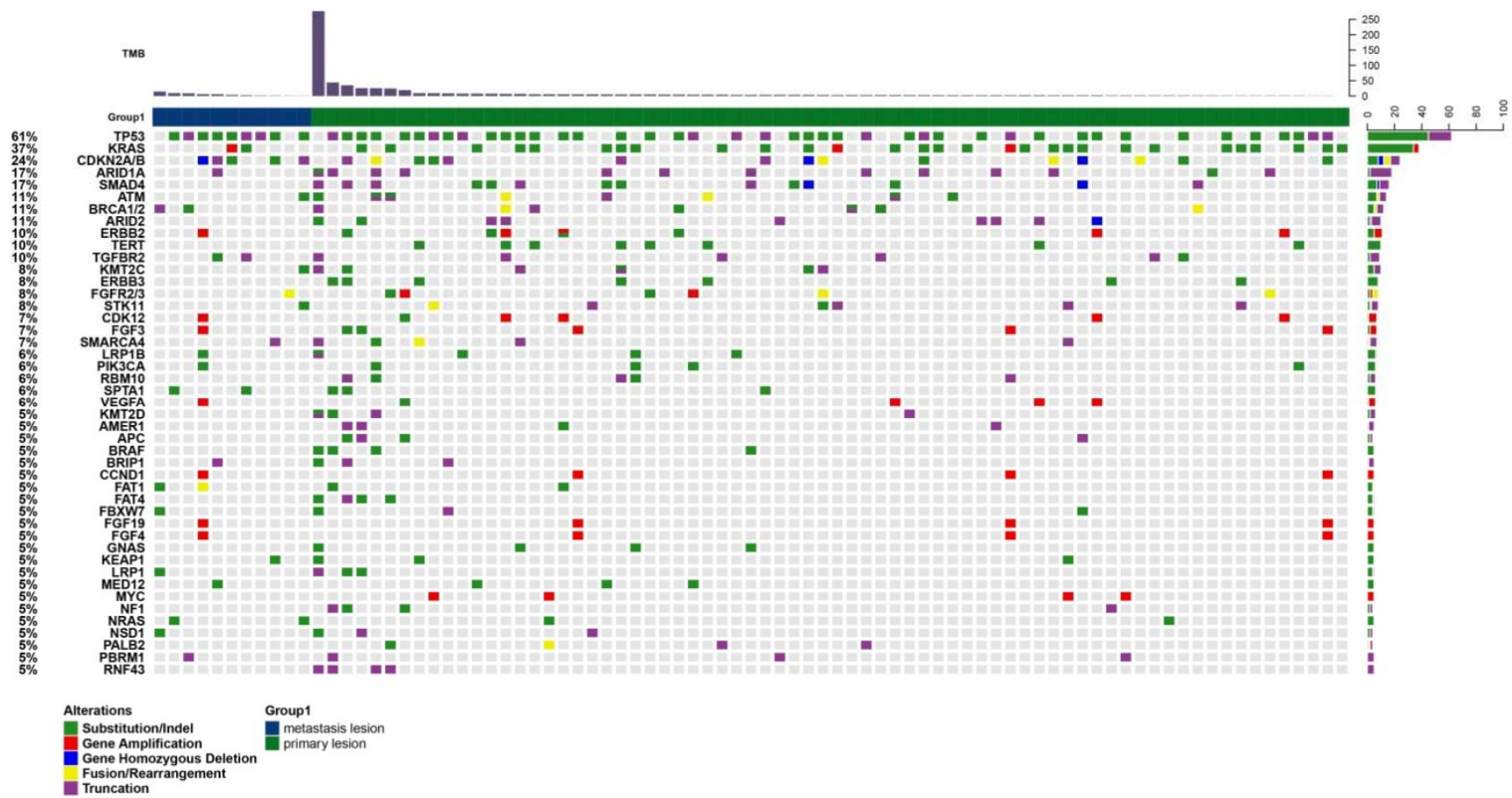

Figure 1. Thermogram of gene sequencing of gallbladder cancer and cholangiocarcinoma. Through comprehensive analysis of heat map, we found that the most common gene mutations in ICCA were TP53, KRAS, ARID1A, CDKN2A, SMAD4 and ERBB; TP53, KRAS, CDKN2A, ARID2, SMAD4 and ARID1A are the most common mutations in ECCA. While TP53, ERBB, CDKN2A, PIK3CA, ARID1A, CCNE1 are the most common mutations in GBC.

We found that the mutation rate of TP53 gene are $38.09 \%$ in ICCA, $48.97 \%$ in ECCA and $70 \%$ in GBC. Of which $28 \%$ are arginine mutations. It suggests that TP53 gene mutation plays an important role in the pathogenesis of CCA and
GBC. TP53 gene is the most common mutation of tumor suppressor gene in human malignant tumors. Clinical studies have shown that mutant p53 promotes tumor invasion and metastasis through TGF-beta receptor, epidermal growth 
factor receptor and MET signal [5]. Mutations of TP53 gene can affect the occurrence, progression and therapeutic effect of cancer. More than $90 \%$ of TP53 gene mutations are located in the core domain, and the function of most p53 protein isomers will be affected by mutations. The core domain contains DNA binding region. Almost every amino acid site in this region is associated with cancer [6]. At present, there is no FDA approval for targeted anti-cancer drugs for TP53 mutation. WEE1 inhibitors AZD1775 and SGT-53 have been tested in solid tumors [7]. Another drug, APR-246, can stabilize the conformation of mutant p53 protein and increase the sensitivity of cancer cells to chemotherapy [8].

RAS genes (including NRAS, KRAS and HRAS) are common mutations in human tumors. The mutation rates of KRAS gene in ICCA, ECCA and GBC are $33.33 \%, 51.02 \%$ and $7.5 \%$, respectively. We found that $70 \%$ of them are glycine mutations in the 12 encoding. These mutations can affect the hydrolysis of GTP mediated by RAS, and lead to persistent activation of RAS, which promotes the development of CCA and GBC [9]. KRAS gene encodes a RAS protein with GTPase activity, which is the key regulatory protein of EGFR signaling pathway. It can promote cell proliferation, migration, survival, angiogenesis, metabolism and immune escape [10]. At present, it is generally believed that KRAS gene mutation is a poor prognostic factor for many cancers [11]. Cancer caused by KRAS gene mutation is reported to respond to MEK inhibitors [12]. A phase I clinical trial of solid tumors showed that patients with KRAS mutation reduced their tumors by $6 \%$ to $52 \%$ after taking Trimetinib [13]. Phase I/II clinical trials of piperacilli (CDK4/6 inhibitor) combined with PD0325901 (MEK inhibitor) in the treatment of KRAS mutant solid tumors are under way (NCT02022982) [14].

After our investigation, we found that 25 out of 91 patients with cholangiocarcinoma were positive for PD-L1. In addition, 18 of 25 patients had TP53 and KRAS gene mutations, accounting for $72 \%$, which indicated that TP53 and KRAS gene mutations were related to the positive expression of PD-L1, and the above two gene mutations had a certain impact on tumor immune microenvironment.

ARID1A is a cancer suppressor gene with ATPase activity, which regulates the target gene by changing the structure of chromosomes around the target gene. The mutation rate of ARID1A gene in ICCA, ECCA and GBC are $28.57 \%, 8.16 \%$ and $15 \%$, respectively. Most of the mutate is termination codons. ARID1A gene mutations in tumors are nonsense mutations or frameshift mutations, which lead to loss of protein expression [15]. The deletion of ARID1A gene leads to the activation of PI3K/Akt pathway and increases the phosphorylation level of Akt [16]. DNA repair defects occur in ARID1A-deficient cancer cells, which are prone to DNA damage [17]. In ICCA, low expression of ARID1A protein is associated with poor prognosis. Inhibition of ARID1A protein expression could induce hepatocellular carcinoma in mice [18]. At present, there is no FDA approved anti-cancer drug targeting
ARID1A gene. A phase II clinical trial with olapali for the treatment of tumors is being recruited (NCT02576444, OLAPCO), in which Orapali and Akt inhibitor AZD5363 were used in combination.

The mutation of CDKN2A gene was related to many malignant tumors. The mutation rate of CDKN2A gene in ICCA, ECCA and GBC are 19.04\%, 16.32\% and 22.5\%, respectively. Of which $29 \%$ are the decrease of gene copy number. CDKN2A produced two kinds of proteins P14 and p16 through selective splicing. P14 inhibited the degradation of TP53 by interacting with MDM2 [19]. Deletion of CDKN2A allele is associated with worse progression-free survival $(\mathrm{P}<0.001)[20]$. Pabosini and Rebosini are FDAapproved oral CDK4/6 inhibitors. At present, there is no clinical trial on the application of reboxinib in patients with cholangiocarcinoma. In addition, AKT inhibitor MK2206 is currently being studied for the treatment of advanced refractory cholangiocarcinoma (NCT01425879).

The mutation rate of ERBB gene in ICCA, ECCA and GBC are $16.66 \%, 8.16 \%$ and $25 \%$, respectively. $69 \%$ of them are gene amplification. ERBB belongs to the human epidermal growth factor receptor family, which includes four Members: EGFR, HER2, HER3 and HER4; they are transmembrane receptor tyrosine kinases, involved in the growth factor mediated carcinogenic signal cascade amplification and transmission. Studies have shown that continued expression of HER2 in the gallbladder epithelium can lead to adenocarcinoma, and patients with overexpression have a worse 5-year overall survival [21]. The clinical experimental study showed that Lapatinib combined with Gemcitabine could inhibit the proliferation of gallbladder cancer cell line (TGBC1-TKB) in cooperation [22].

Other common mutations are SMAD4, ARID2, ATM, CDK12, CCNE1. Among the ARID2 mutations, 54\% were glutamine mutations. We analyzed that the mutation can cause the degradation of mRNA and result in the loss of protein expression. In the mutations of CDK12 gene, $80 \%$ were gene amplification. It is associated with malignant transformation and progression of pleomorphic adenoma. The variation rate of CCEN1 gene in CCA and GBC are $9.18 \%$ and $15 \%$, all of which showed the increase of gene copy number. The amplification of this gene is related to the prognosis of many tumors. Over expression of CCNE1 can increase the resistance of HCC to Sorafenib. A phase II clinical trial using Wee1 inhibitor AZD1775 to treat solid tumor patients with CCNE1 gene amplification has been initiated (NCT03253679).

In addition, we also found that in CCA and GBC, the mutation of TERT gene all changed from cysteine to threonine, which was 124 bases upstream of translation initiation codon. All mutations of GNAS gene occur in ICCA, and the mutation site are arginine mutation to histidine in 201 Codon. Some datas show that progressionfree survival of patients with high expression of GNAS is significantly shorter than that of patients with low expression, suggesting that over expression of GNAS is related to prognosis [23]. 


\section{Clinical Analysis of PD-1 Inhibitor in the Treatment of CCA}

Immune inhibitors of programmed death receptor 1 (PD1) and programmed death receptor ligand 1 (PDL-1) have become a hotspot in the field of cancer therapy. Because of their long-lasting antineoplastic efficacy and wide applicability, they have become the most promising and therapeutic strategy for cancer immunotherapy. PD-1 is mainly expressed in activated $\mathrm{T}$ cells and $\mathrm{B}$ cells, and its function is to inhibit the activation of cells, which is a normal homeostasis mechanism of the immune system [10]. PD-L1 is up-regulated in a variety of tumor cells. It combines with PD-1, inhibits the proliferation and activation of $\mathrm{T}$ cells, makes it inactive, and finally induces tumor immune escape. Therefore, immunotherapy targeting PD-1 and PD-L1 can kill tumor cells by activating antitumor immunity.

According to the inclusion criteria, we chose patients who could not resect the tumor or relapse after surgery for PD-1 inhibitor treatment. After NGS test, there are 25 patients with PDL-1 positive or TMB medium and high (Table 3). According to the sequence of tumor species, TMB was divided into high, medium and low. TMB in the $0 \%-25 \%$ range is high, $26 \%-75 \%$ is medium, $76 \%-100 \%$ is low. The higher TMB, the better effect of PD-1 inhibitor (Figure 2). According to clinical guidelines, PD-1 inhibitors can only be used PDL-1 positive or TMB medium and high.

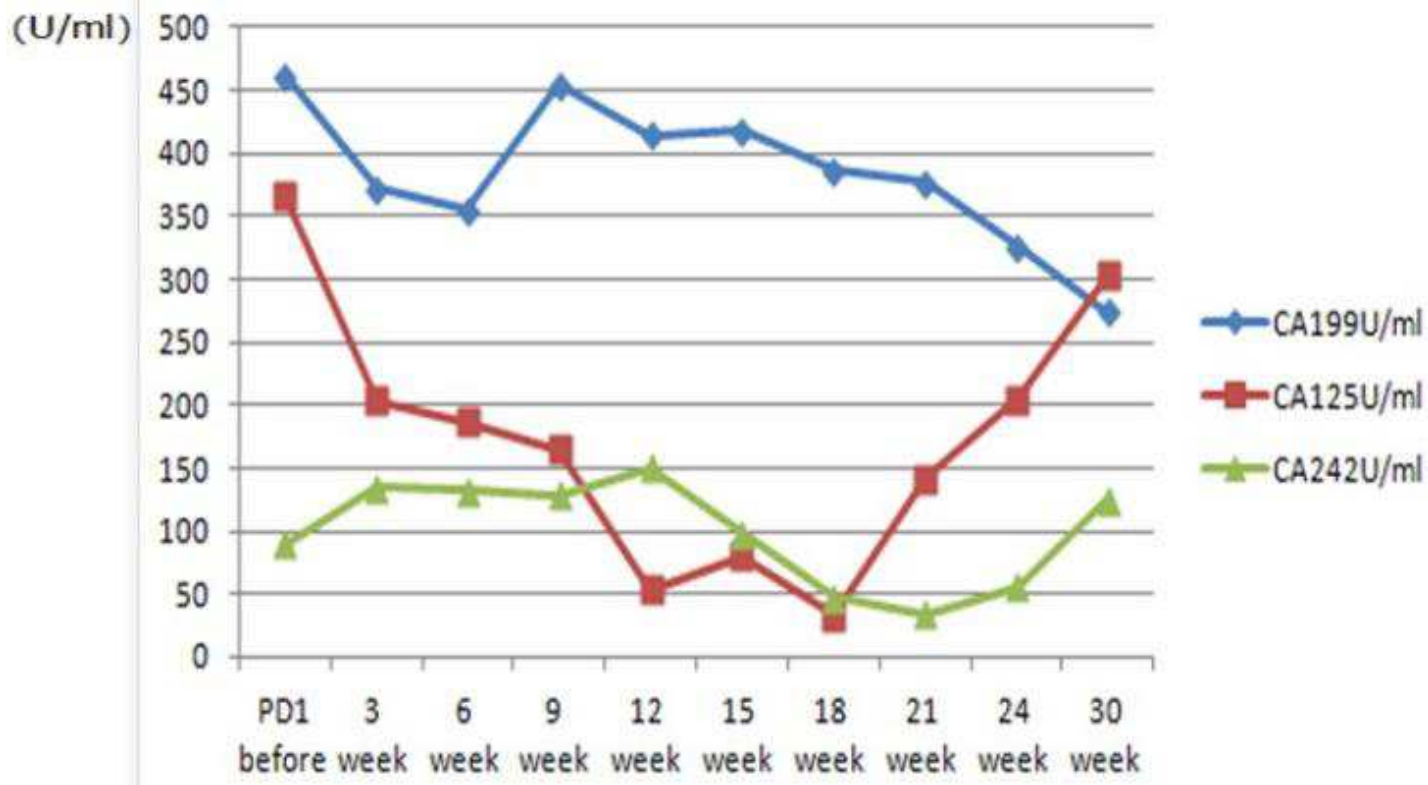

Figure 2. Data of CA199, CA125, CA242 mean changes before and after PD-1 inhibitors. It was found that the tumor markers of most patients decreased to some extent after PD1 inhibitor treatment, but after a period of treatment, the tumor markers of some patients increased again, indicating that these patients had drug resistance to PD1 inhibitor.

Table 3. Comparison of MSI, PDL-1 and TMB in ICCA, ECCA and GBC.

\begin{tabular}{|c|c|c|c|c|}
\hline & $\operatorname{ECCA}(n=49)$ & $\operatorname{ICCA}(n=42)$ & GBC $(n=40)$ & $\operatorname{ALL}(\mathrm{N}=131)$ \\
\hline MSI-H & $1(2 . .04 \%)$ & $3(7.14 \%)$ & $2(5 \%)$ & $6(4.58 \%)$ \\
\hline PDL-1 positive & $5(10.2 \%)$ & $11(26.19 \%)$ & $8(20 \%)$ & $24(18.32 \%)$ \\
\hline Low TMB & $36(73.46 \%)$ & $30(71.42 \%)$ & $18(45 \%)$ & $84(64.12 \%)$ \\
\hline High TMB & $3(6.12 \%)$ & $4(9.52 \%)$ & $4(10 \%)$ & $11(8.39 \%)$ \\
\hline
\end{tabular}

MSI-H: microsatellite instability-high. There is a certain correlation between tissue MSI test score and immunotherapy. TMB: Tumor mutation burden. It refers to the number of somatic mutations per million bases in the coding region of targeted sequencing, including point mutation and insertion deletion. Low TMB (1-5 mutations / MB), Medium TMB (6-19 mutations / MB), High TMB ( $\geq 20$ mutations / MB). PDL-1: Interaction between PD-L1 and its receptor PD1 can inhibit the activation of $\mathrm{T}$ cells and the production of cytokines, providing an immune escape mechanism for tumor cells.

Through gene testing, 15 patients use Pembrolizumab and 10 patients use nivolumab. Drugs are given every two or three weeks. By follow-up, about 6 weeks later, 15 patients CA19-9, CA125, CA242 tumor markers decreased to some extent, which also prolonged the progression free survival and overall survival (Figure 2). It has been reported that the average survival rate of CCA is 9 months [24]. However, after follow-up, the objective response rate of patients with
PD-1 inhibitors was 24\%. The Progression free survival (months) of the patients are $5.2 \pm 4.8$ months and the longest Progression free survival (months) up to 17 months. The Overall survival (months) of patients with PD-1 inhibitors is $11.9 \pm 5.9$ months. No patient has been completely relieved. 6 patients had partial remission and 13 patients stabled after using PD-1 inhibitor. In addition, some patients were found that the diameter of the tumor significantly smaller than 
before treatment (Figure 3). Although PD-1 inhibitors showed ideal anti-tumor effects in many solid tumors, but not all patients could benefit from this treatment. We also found 6 patients did not benefit from the treatment. Their condition did not improve and the tumor continuous increase. However, it is worth noting that these patients have severe pain caused by tumor. They need to use analgesics every day. Although the survival period of patients after using PD-1 inhibitors has not been extended, but their pain symptoms have been well and generally relieved. It can be proved that PD-1 inhibitor has a good effect of inhibiting pain caused by tumor, and the specific mechanism needs further study.

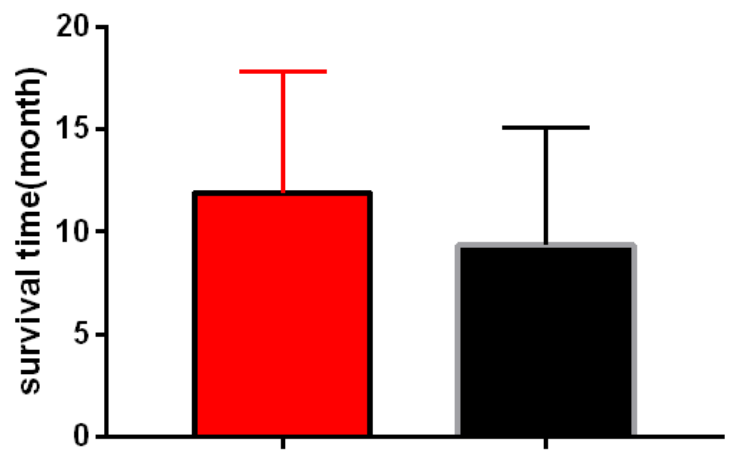

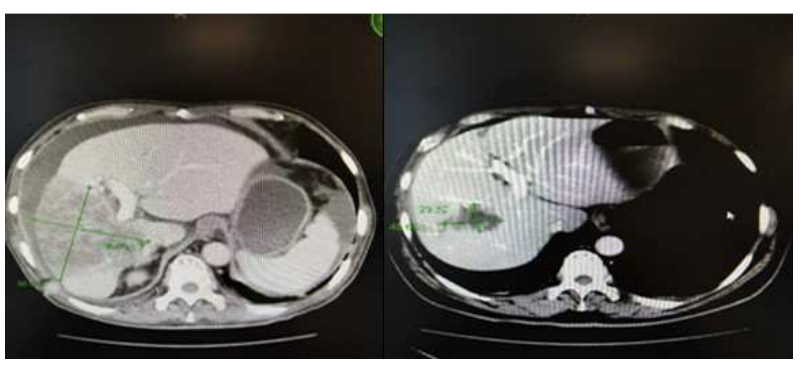

Figure 3. Contrast CT image before and after using PD-1 inhibitor. Before treatment, the size of ICCA was about $98 \mathrm{~mm} * 96 \mathrm{~mm}$. After 2 months of PDI inhibitor treatment, the size of tumor was significantly reduced to $43 \mathrm{~mm} * 29$ $m m$.

\section{Overall survival}

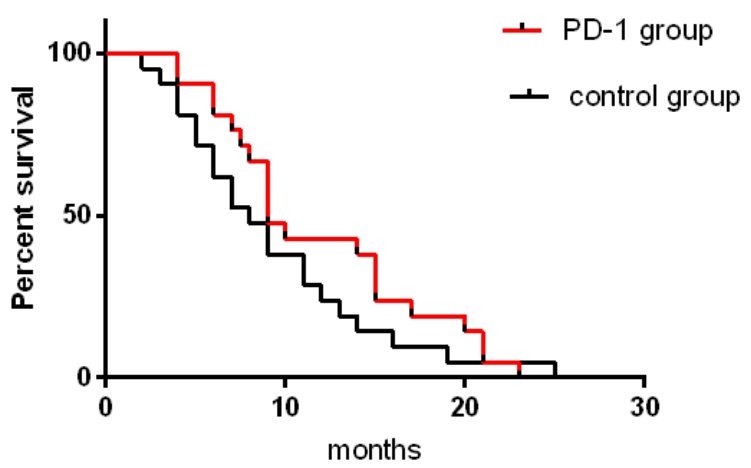

Figure 4. Comparison of overall survival rate between the two groups. We found that the overall survival rate was slightly longer in patients with PDI inhibitors.

\section{Conclusion}

Transforming NGS into oncology practice promotes the development of precision medicine, which tailors anti-cancer programs for everyone according to gene maps. Compared with traditional treatments focusing on the primary site of tumors, precision medicine is dedicated to identifying operable genetic changes. Gene changes vary widely among individuals, partly reflecting the heterogeneity of tumors, which may be related to the specific environment [25]. Sequencing multiple tumor sites at different time and matching identified operational targets may be the best method. Fortunately, NGS provides an opportunity to identify mutated genes. Through the observation of patients using PD-1 inhibitor for nearly half a year, we found that the progression free survival period and overall survival rate of patients were prolonged. After a follow-up of 25 patients with CCA treated with PD-1 inhibitor, we found the objective remission rate reached $60 \%$. Although all patients have indications of using PD-1, the complete failure rate is still $40 \%$. Our study found that the higher the TMB, the better the effect on PD-1 inhibitor. Three patients have been using PD1 inhibitor for 10 months to one year, and their condition is still in a stable state. In all of these patients, the codon 12 of KRAS gene mutation was changed from glycine to valine. However, CDKN2A and PREX2 gene mutation are more common among PD-1 inhibitor insensitive patients. CDKN2A gene encodes two different and unrelated tumor suppressor proteins, p16INK4a and p14ARF. P16INK4a can bind and inhibit the expression of CDK4 and CDK6, so as to maintain the function of tumor suppressor RB. P14ARF can regulate p53 mediated apoptosis by inhibiting the stability and activation of MDM2 [26]. PREX2 may play a role in carcinogenesis by inhibiting phosphatase activity and activating phosphoinositide kinase signaling pathway [27]. We consider that mutations of the above two genes may lead to PD-1 inhibitors resistance and reduce the efficacy, but it still needs to be confirmed by research.

\section{Availability of Data and Materials}

The datasets used and analyzed during the current study are available from the corresponding author on reasonable request.

\section{Authors' Contributions}

Feiling Feng and Xiaoqing Jiang designed the study; Hao Song performed data collection, data analysis, collected the clinical data, prepared the manuscript and approved the publication of the final version.

\section{Ethics Approval and Consent to Participate}

This study was approved by Ethics Committee of the 
Oriental Hepatobiliary Surgery Hospital affiliated to the Second Military Medical University (Shanghai, China).

\section{Competing Interests}

The authors declare that they have no competing interests.

\section{Acknowledgements}

Not applicable.

\section{References}

[1] Tan JC, Coburn NG, Baxter NN, et al. Surgical management of intrahepatic cholangiocarcinoma--a population-based study. Ann Surg Oncol. 2008 (15): 600-608.

[2] Khan SA, Davidson BR, Goldin RD, et al. Guidelines for the diagnosis and treatment of cholangiocarcinoma. Gut. 2012 (61): 1657-69.

[3] Torre LA, Bray F, Siegel RL, et al. Global cancer statistics, 2012. CA Cancer J Clin. 2015 (65): 87-108.

[4] Grugan KD, Vega ME, Wong GS, et al. A common p53 mutation $(\mathrm{R} 175 \mathrm{H})$ activates c-Met receptor tyrosine kinase to enhance tumor cell invasion. Cancer Biol Ther. 2013, 14 (9): 853-9. PMID: 2379258.

[5] Wang W, Cheng B, Miao L, et al. Mutant p53-R273H gains new function in sustained activation of EGFR signaling via suppressing MIR-27a expression. Cell Death Dis. 2013, 4: 574. PMID: 23559009.

[6] Leroy B, Anderson M, Soussi T. TP53 mutations in human cancer: database reassessment and prospects for the next decade. Hum Mutat. 2014, 35 (6): 672-88. PMID: 24665023.

[7] Senzer N, Nemunaitis J, Nemunaitis D, et al. Phase I study of a systemically delivered $\mathrm{p} 53$ nanoparticle in advanced solid tumors. Mol Ther. 21 (5), 1096-103. (2013). PMID: 23609015.

[8] Necchi A, Lo Vullo S, Mariani L, et al. An open-label, singlearm, phase 2 study of the Aurora kinase A inhibitor alisertib in patients with advanced urothelial cancer. Invest New Drugs. 34 (2), 236-42. (2016). PMID: 26873642.

[9] Valtorta E, Misale S, Sartore-Bianchi A. KRAS gene amplification in colorectal cancer and impact on response to EGFR-targeted therapy. Int J Cancer. 2013, 133 (5): 1259-65. PMID: 23404247.

[10] Kato S1, Goodman A2, Walavalkar V3, et al. Hyperprogressors after Immunotherapy: Analysis of Genomic Alterations Associated with Accelerated Growth Rate. Clin Cancer Res. 2017, 23 (15): 4242-4250. PMID: 28351930.

[11] Chung WC, Wang J, Zhou Y, et al. KrasG12D upregulates Notch signaling to induce gallbladder tumorigenesis in mice. Oncoscience. 2017, 4 (9-10): 131-138. PMID: 29142904.

[12] Bendell JC, Javle M, Bekaii-Saab TS, et al. A phase 1 doseescalation and expansion study of binimetinib (MEK162), a potent and selective oral MEK1/2 inhibitor. Br J Cancer. 2017. PMID: 28152546.
[13] Robert, C, Long, G, Brady, et al. Nivolumab in previously untreated melanoma without BRAF mutation. N Engl J Med. 2015. PMID: 25399552.

[14] Engelman JA, Chen L, Tan X. Effective use of PI3K and MEK inhibitors to treat mutant Kras G12D and PIK3CA H1047R murine lung cancers. Nat Med. 2018, 14 (12), 135156. PMID: 19029981.

[15] Bitler BG, Fatkhutdinov N, Zhang R. Potential therapeutic targets in ARID1A-mutated cancers. Expert Opin Ther Targets. 2015, 19 (11): 1419-22. PMID: 26125128.

[16] Chandler RL, Damrauer JS, Raab JR, et al. Coexistent ARID1A-PIK3CA mutations promote ovarian clear-cell tumorigenesis through pro-tumorigenic inflammatory cytokine signalling. Nat Commun. 2015, 6: 6118. PMID: 2562562582.

[17] Watanabe R, Ui A, Kanno S, Ogiwara H, et al. SWI/SNF factors required for cellular resistance to DNA damage include ARID1A and ARID1B and show interdependent protein stability. Cancer Res. 2014, 74 (9): 2465-75. PMID: 24788099.

[18] Fang JZ, Li C, Liu XY, et al. Hepatocyte-Specific Arid1a Deficiency Initiates Mouse Steatohepatitis and Hepatocellular Carcinoma. PLoS One. 2015, 10(11): 1430-42. PMID: 26569409.

[19] Ruas M, Peters G. The p16INK4a/CDKN2A tumor suppressor and its relatives. Biochim Biophys Acta. 1998, 1378 (2): 115119. PMID: 9823374.

[20] Andrew H. Girgis. Clear Cell Renal Cell Carcinoma With Biallelic Inactivation Of CDKN2A/B On 9p21 Have Distinct Gene Expression Signature And Are Associated With Poor Prognosis. BioRxiv 2017. PMID: AB000220.

[21] Roa I, de Toro G, Schalper K, et al. Over expression of the HER2/neu Gene: A New Therapeutic Possibility for Patients with Advanced Gallbladder Cancer. Gastrointest Cancer Res. 7 (2), 42-8. (2014). PMID: 24799970.

[22] Pignochino Y, Sarotto I, Peraldo-Neia C, et al. Targeting EGFR/HER2 pathways enhances the antiproliferative effect of gemcitabine in biliary tract and gallbladder carcinomas. BMC Cancer. 10, 631. (2010). PMID: 21087480.

[23] Gibbons GS1, Owens SR, Fearon ER, Nikolovska-Coleska Z. Regulation of Wnt signaling target gene expression by the histone methyltransferase DOT1L. ACS Chem Biol. 2015, 10(1): 109-114. DOI: 10.1021/cb500668u.

[24] Launonis B, Terblanche J, Lakehal M, et al. proximal bile duct cancer: high respectabiliy rate and 5 year survival. Ann Surg, 1999, 230 (2): 266

[25] Burrell RA, McGranahan N, Bartek J, Swanton C. The causes and consequences of genetic heterogeneity in cancer evolution. Nature. 2013; 501: 338-345.

[26] Ozenne, P., Eymin, B., Brambilla, E. et al. The ARF tumor suppressor: structure, functions and status in cancer. Int $\mathrm{J}$ Cancer. 2010. DOI: 10.1002/ijc.25511.

[27] Dai Z, Xie J, Lei P. Knockdown of PREX2a inhibits the malignant phenotype of osteosarcoma cells. Mol Med Rep. 2016, 13: 1930-1936. 